Automatic guided wave PPM communication system for potential SHM of flooding members in sub-sea oilrigs

This article has been downloaded from IOPscience. Please scroll down to see the full text article.

2013 Smart Mater. Struct. 22055031

(http://iopscience.iop.org/0964-1726/22/5/055031)

View the table of contents for this issue, or go to the journal homepage for more

Download details:

IP Address: 130.88.154.16

The article was downloaded on 22/04/2013 at 17:01

Please note that terms and conditions apply. 


\title{
Automatic guided wave PPM communication system for potential SHM of flooding members in sub-sea oilrigs
}

\author{
Rito Mijarez ${ }^{1}$ and Patrick Gaydecki ${ }^{2}$ \\ ${ }^{1}$ Gerencia de Control, Electrónica y Comunicaciones, Instituto de Investigaciones Eléctricas, 62490, \\ Cuernavaca, Morelos, Mexico \\ ${ }^{2}$ School of Electrical and Electronic Engineering, The University of Manchester, PO Box 88, \\ Manchester M60 1QD, UK \\ E-mail: rmijarez@iie.org.mx
}

Received 2 January 2013, in final form 7 January 2013

Published 18 April 2013

Online at stacks.iop.org/SMS/22/055031

\begin{abstract}
An automatic guided wave pulse position modulation system, using steel tubes as the communication channel, for detecting flooding in the hollow sub-sea structures of newly built offshore oilrigs is presented. Underwater close visual inspections (CVI) are normally conducted during swim-round surveys in pre-selected areas or areas suspected of damage. An acceptable alternative to CVI is a non-destructive testing (NDT) technique called flood member detection (FMD). Usually, this NDT technique employs ultrasound or x-rays to detect the presence of seawater in the tubular structures, requiring divers or remote operating vehicles (ROVs). The field-proven FMD technique, integrated within the concept of structural health monitoring, offers an alternative to these traditional inspection methods. The system employs two smart sensors and modulators, which transmit $40 \mathrm{kHz}$ guided wave pulses, and a digital signal processing demodulator, which performs automatic detection of guided wave energy packets. Experiments were performed in dry conditions, inside and outside the laboratory; in the former using a steel tube $1.5 \mathrm{~m} \times 0.27 \mathrm{~m} \times 2 \mathrm{~mm}$, and in the latter using a tubular steel heliport structure approximately $15 \mathrm{~m} \times 15 \mathrm{~m}$ in area and the base deck of an oilrig under construction. Results confirm that, although there was significant dispersion of the transmitted pulses, the system successfully distinguished automatically guided wave encoded information that could potentially be used in sub-sea oilrigs.
\end{abstract}

(Some figures may appear in colour only in the online journal)

\section{Introduction}

Structural health monitoring (SHM) is a growing technology that includes systems for detecting and interpreting adverse changes in a structure to improve reliability and reduce lifecycle costs, by eliminating periodic inspections, preventing underuse and overuse, and predicting failure in time to prevent hazard [1, 2]. Non-destructive testing (NDT) techniques, when included within the conceptual framework of SHM, offer encouraging and challenging possibilities to facilitate quick and accurate automated damage detection [3]. The principal building blocks of an SHM technology are sensors, data acquisition hardware and a communication platform; sensors are embedded in or mounted permanently on the surface of a structure and provide feedback on mechanical integrity through analysis of measurable data. Novel wireless communication channels are a necessity in harsh environments, for instance fixed offshore oilrigs and down-hole oil reservoirs. In these applications the environmental conditions are often very severe, and the use of cables dedicated exclusively as communication media has proved to be impractical $[4,5]$.

Regarding fixed offshore oilrigs, inspections focus on areas susceptible to cracking to determine if damage has 
occurred, for example, fatigue sensitive joints and the health of underwater supporting steel brace structures, normally made from sealed steel tubes filled with air. Hence it is important to have information on the ultimate strength of tubular brace members, with circumferential cracks, to determine their reliability before severance can occur. The ingress of seawater via through-thickness cracks into these sealed crossbeams is a crucial issue, and is used as a basis for the development of the inspection method known as flood member detection (FMD), provided that the member is able to tolerate the presence of cracks of through-thickness dimensions without making the integrity of the structure unacceptable [6]. The FMD technique has been used for some considerable time, with NDT underwater ultrasonic probes or gamma radiation sources. However, these schedule-driven inspections are expensive to hire and operate, requiring the deployment of a diver or a ROV [4].

The combination of a field-proven underwater NDT techniques, such as FMD, within the context of SHM, has been proposed by the authors [7]. It provides an attractive technology that is capable of continuously and autonomously monitoring for damage to the supporting sub-sea steel structures in offshore platforms. This may favourably be applied to overcoming the deficiencies of traditional FMD by eliminating inspection intervals, providing early warning of failure during operation, and bringing about life-cycle cost reduction. In this alternative, the authors have developed a system that employs a single PZT transducer, which is permanently attached to the inner wall of the lower end of a given sub-sea crossbeam, and is powered by a (normally inert) seawater battery. Upon activation, the sensor transmits ultrasonic chirp or tone encoded pulses, in the range of $21-42 \mathrm{kHz}$, to a monitoring receiver system at deck level for decoding and identifying flooded members. The system exploits the steel structure as a communication channel, which derives benefit from guided waves that propagate through the tubular steel structure. The authors have successfully excited and identified guided wave signals from steel pipes $7 \mathrm{~m}$ in length, $0.5 \mathrm{~m}$ in diameter and $16 \mathrm{~mm}$ in thickness, flooded and immersed in seawater. However, the identification of the signals with sufficient signal to noise ratio (SNR) has been performed visually in the monitoring receiver at surface level $[7,8]$.

This work presents the initial results obtained from an automatic pulse position modulation (PPM) system that could be used for SHM of tubular members in fixed offshore oilrigs, innovatively using the tubular structure as a communication channel. The system transmits $40 \mathrm{kHz}$ ultrasonic guided waves pulses through hollow steel tubes. The system employs two smart transmitter modulators composed of a PZT element, electronics and batteries, and demodulator instrumentation that is made of a PZT ultrasound transducer, a real-time digital signal processing (DSP) board and a PPM module based on a microcontroller, which performs automatic PPM detection of guided wave energy packets. Experiments carried out in air in the laboratory using a steel tube of $1 \mathrm{~m}$ length, and outside the laboratory in a tubular steel heliport structure and the base of a deck of an oilrig under construction, have successfully detected automatically guided wave PPM information. These results form the basis for developing smart sensors that can be embedded inside crossbeam members of an oilrig under construction for automatic monitoring purposes.

\section{SHM and guided waves}

The exploitation of guided ultrasonic waves in the context of SHM is becoming more commonplace in industry $[9,10]$. Of particular interest is its use in the monitoring of pipes, in particular, pipes either evacuated or filled and surrounded by water. This provides the possibility of using guided waves for FMD, by leaving in place transducers capable of transmitting information from the internal part of the previously hollow sub-sea structure of an in-service offshore installation to monitoring systems at deck level. The receiving transducer is attached to the external and dry part of the steel tubular platform, above the water level. In this work, hollow steel tubes, in air, have been considered as a communication channel for preliminary testing the automatic detection of guided wave pulses. This section deals with the theoretical background of using the steel pipe as a communication medium, the selection of transducers, frequency and signals.

\subsection{Theoretical background}

The frequency equation for wave propagation in a hollow isotropic elastic cylinder has been derived in detail by Gazis [11]. In general, there are three principal types of guided wave modes that can exist in a cylindrical waveguide: the longitudinal (or $L(0, m)$ ), the torsional (or $T(0, m)$ ) and the flexural (or $F(M, m)$ ). In theory, there are an infinite number of individual modes within each principal mode, whose phase velocities, $V_{\mathrm{ph}}$, for a given frequency-tube thickness product, $f_{d}$, represent permissible solutions to an implicit transcendental equation of the form

$$
\Omega_{M}\left(a, b, \lambda, \mu, f_{d}, V_{\mathrm{ph}}\right)=0
$$

where $a$ and $b$ stand for the inner and outer radii of the tube and $\lambda$ and $\mu$ represent its Lame constants. The index $M$ determines the manner in which the fields generated by the guided wave modes vary with the angular coordinate $\theta$ in the cylinder cross-section. Each field component can be considered to vary as

$$
\begin{aligned}
& u_{r}=U_{r}(r) \cos M \theta \cos (\omega t+k z), \\
& u_{\theta}=U_{\theta}(r) \sin M \theta \cos (\omega t+k z), \\
& u_{z}=U_{z}(r) \cos M \theta \sin (\omega t+k z),
\end{aligned}
$$

where the circumferential order $M=0,1,2,3, \ldots$ The terms $u_{r}, u_{\theta}, u_{z}$ are the displacement components in the radial, circumferential and axial directions, respectively; the terms $U_{r}, U_{\theta}, U_{z}$ are the displacement amplitudes composed of Bessel or modified Bessel functions, depending on the argument. All of the modes propagate in the axial direction of a cylindrical waveguide. The acoustic fields (i.e. displacement, stress, etc) of the modes when $M=0$ are axially symmetric along a cylinder circumference and are 


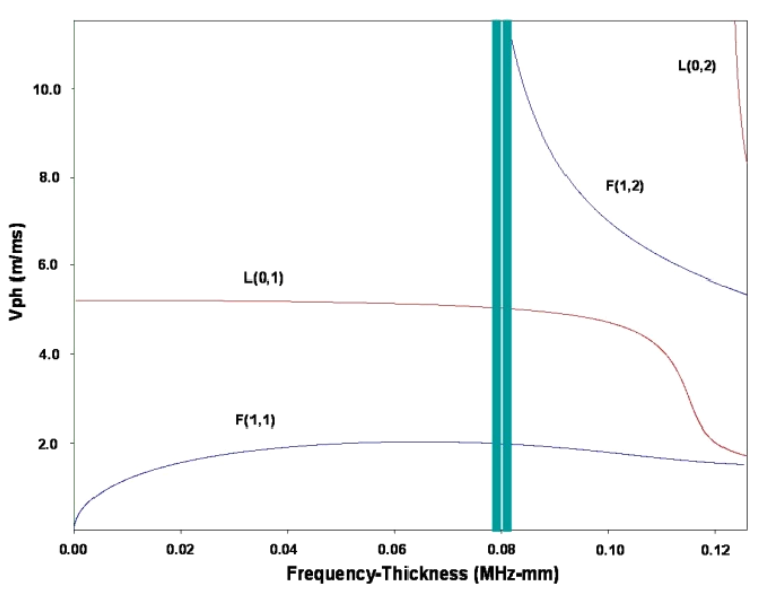

a)

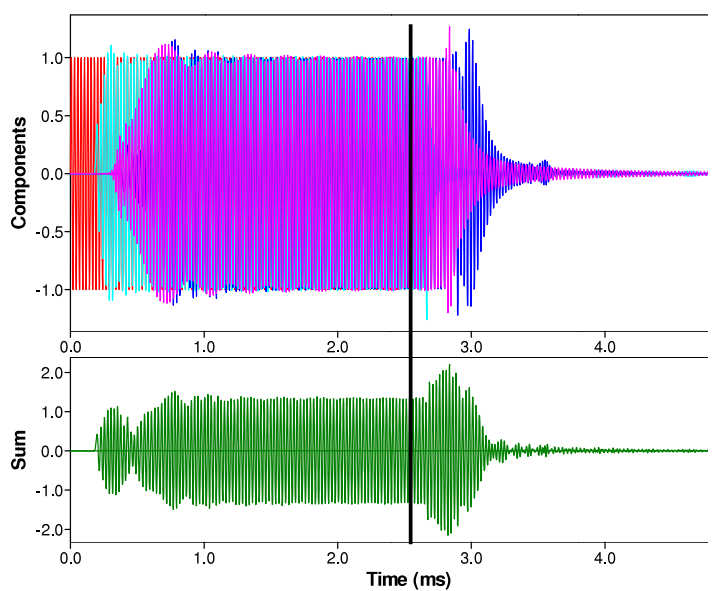

b)

Figure 1. (a) Dispersion curve of a hollow steel tube of $2 \mathrm{~mm}$ thickness and $0.27 \mathrm{~m}$ diameter; (b) multiple modes generated from the transmission point, including $L(0,1), F(1,1)$ and $F(1,2)$.

independent of the angular coordinate $\theta$. The other modes are non-axisymmetric and have fields which do vary with the angular coordinate $\theta$.

The axisymmetric modes comprise both the longitudinal modes, $L(0, n)$, and the torsional modes, $T(0, n)$; the non-axisymmetric modes are represented by the flexural modes, $F(M, n)$ [12]. Longitudinal axially symmetric modes are frequently preferred and more implemented in practical applications than torsional and flexural modes. They offer better experimental aspects, such as ease of excitability and repeatability than torsional modes, and are preferred over flexural modes for excitation owing to the symmetry that allows the inspection through $360^{\circ}$ along the circumference of the tubes. The longitudinal modes are the easiest to generate using conventional wedge type transducers and liquid couplant, compared to the torsional modes, which can only be generated by applying shear forces, their behaviour depends on both the geometry of the tube in which they propagate and the frequency at which they are generated.

Classifications of velocity dispersion curves which characterize the solutions to equation (1) are achieved, pragmatically, in terms of a frequency-thickness product, $f_{d}$, of the waveguide. Figure 1(a) shows the phase velocity dispersion curve of a steel pipe, $2 \mathrm{~mm}$ in thickness and $0.27 \mathrm{~m}$ in diameter, in air, generated by the software Disperse (c) [13]. In these conditions, attenuation of the propagated guided waves packets is considered null. As shown in figure 1(a), it is possible to have several guided wave modes with a single frequency and all of them with different velocities. The total number of guided wave modes for a given $f_{d}$ value is finite and increases with an increase in frequency. Therefore, having a constant thickness, the selection of a low $f_{d}$ value, where the number of guided wave modes is reduced, depends on a low frequency which possesses a large wavelength.

\subsection{Transducer considerations and frequency selection}

Long-range applications using guided waves involve the employment of frequencies below $100 \mathrm{kHz}$ [14]. The selection of a specific point on the dispersion curves depends not only on the frequency spectrum but also on the phase velocity spectrum associated with the transducer source. Generally, an ultrasonic transducer source can excite all the modes which exist within its frequency spectrum; normally, the spectrum becomes narrower for larger transducers [15]. This is of particular interest considering that PZT elements and an ultrasound transducer procedure with normal beam loading and reception, respectively, have been used in this application. Hence, surface pressure loading will excite longitudinal modes and/or flexural modes depending on the applied pressure distributions on the tube surface [16]. Figure 1(b) depicts the possible excited modes at $40 \mathrm{kHz}$ according to the software Disperse. As figure 1(a) indicates, at frequencies beyond $40 \mathrm{kHz}(0.08 \mathrm{MHz} \mathrm{mm})$ the number of the modes increase; hence the selection of the modes becomes difficult. Reducing the frequency of excitation will excite fewer guided wave modes; however, it exposes the overall system to greater risk of audio bandwidth interference, leading to a poor SNR. A compromise between these factors was considered and a transducer with a centre frequency of $40 \mathrm{kHz}$ was selected; therefore, circular PZT elements were powered with tone pulses of $40 \mathrm{kHz}$.

\subsection{Signal selection}

Narrow band signals are often used as excitation for NDT purposes in order to give good signal strength and to avoid dispersion over long propagation distances. Tone pulses of between 5 and 10 cycles modulated by a Hanning or a Gaussian window are frequently employed [17]. Nevertheless, experimental results have revealed that narrow band chirp signals and long tone pulses employed in conjunction with rectangular band-pass filtering yield better SNR for this application [18]. Furthermore, it has been shown that the use of square pulses not only increases the energy of the transmitted signals, thereby improving the SNR, but also reduces the complexity of the signal generation hardware [19]. 


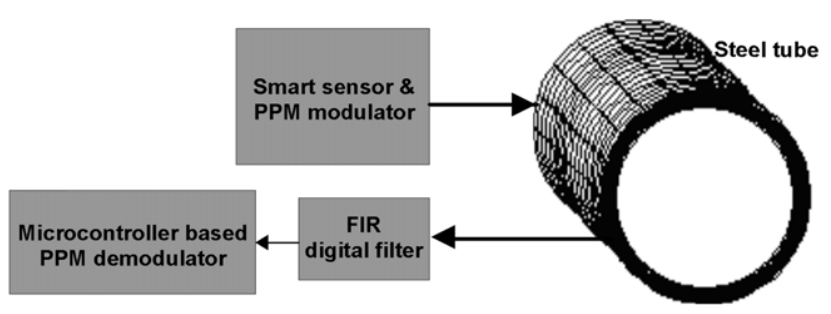

Figure 2. Guided wave PPM modulator and demodulator communication system.

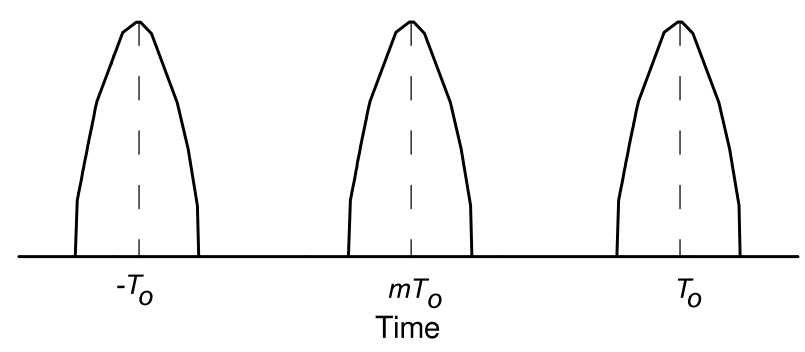

Figure 3. Pulse position modulation soliton.

Moreover, it is not necessary to use digital-to-analogue converters to generate square pulses, and power amplifiers can be substituted by high-speed switching circuits. In this work, hence, square tone pulses of $40 \mathrm{kHz}, 2.5 \mathrm{~ms}$ pulse width were implemented.

\section{PPM guided wave system}

The fundamental research framework of PPM communication systems was established around 50 years ago [20]; PPM has recently also been of interest in the field of impulse radio and fibre-optic transmission systems [21]. PPM is a form of signal modulation in which the message information is modulated through variable time delays between pulses in a sequence of signal pulses. This method, as opposed to pulse width modulation (PWM), is more power efficient, since PWM employs long pulses, which consumes considerable power, and does not offer additional information [22]. Besides, it has been demonstrated theoretically that PPM systems are effective when the signals are power limited rather than band limited [20]. Moreover, the electronics required to demodulate the PPM signals are simple, which leads to small, light-weight receiver/decoder units. Figure 2 depicts a block diagram of a guided wave modulator and demodulator PPM system. In general, the ideal PPM generation of signals, $s_{m}(t)$, is given by [23]:

$$
s_{m}(t)=\varphi\left(t-m T_{0}\right) ; \quad-1 \leq m \leq+1
$$

where $\varphi(t)$ is a unity energy pulse similar to those found in the sampling theorem, and $T_{0}$ is the time delay between pulses (TDBP). The transmitted energy is independent of the value $m$, as depicted in figure 3. In this application, the PPM modulator generates pulses of constant amplitude and predefined TDBP, where the temporal positions of the pulses or TDBP convey the signal information. Different TDBP represents different transmitters, as depicted in figure 4.

\subsection{Transmitter design}

The design and implementation of the PPM sensors and modulators was based on a microcontroller, a current booster, a transformer, a single PZT element and a $9 \mathrm{~V}$ battery, as shown in figure 5(a). The modulator was mounted in a vinyl circular case that incorporated strong rare earth magnets in its

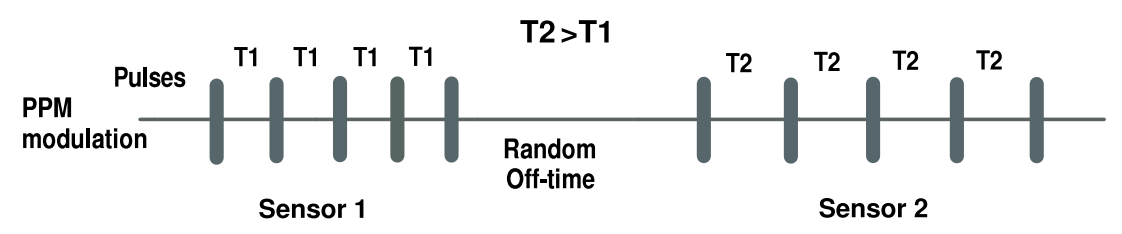

Figure 4. PPM codification, a different temporal position of the pulses (TDBP) represents different transmitters.

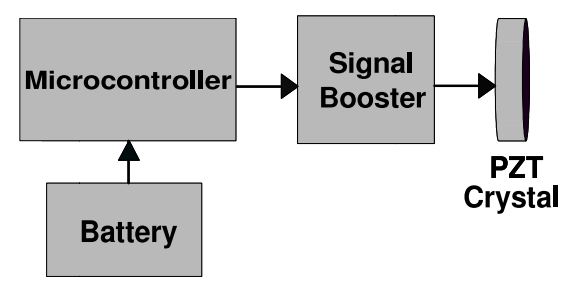

a)

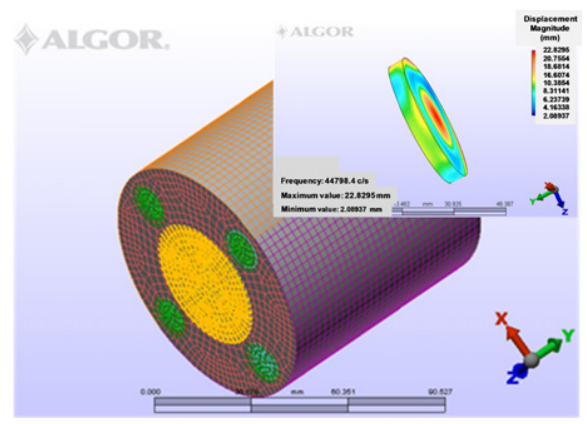

b)

Figure 5. PPM modulator (a) main components; (b) FE model of the vinyl case, magnets and the PZT crystal. 

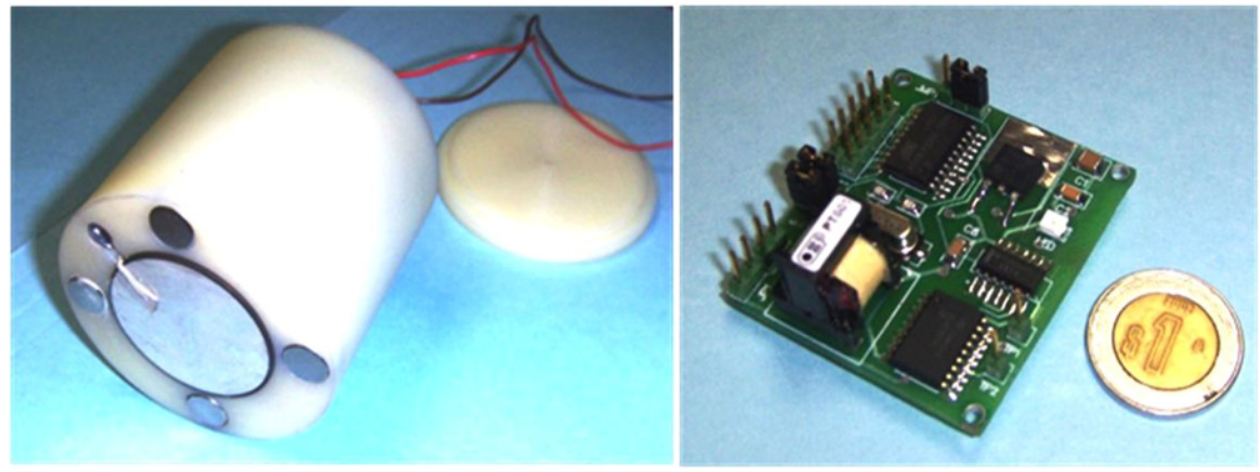

Figure 6. PPM modulator actual design; vinyl case and PZT crystal; electronics fitted into the case.

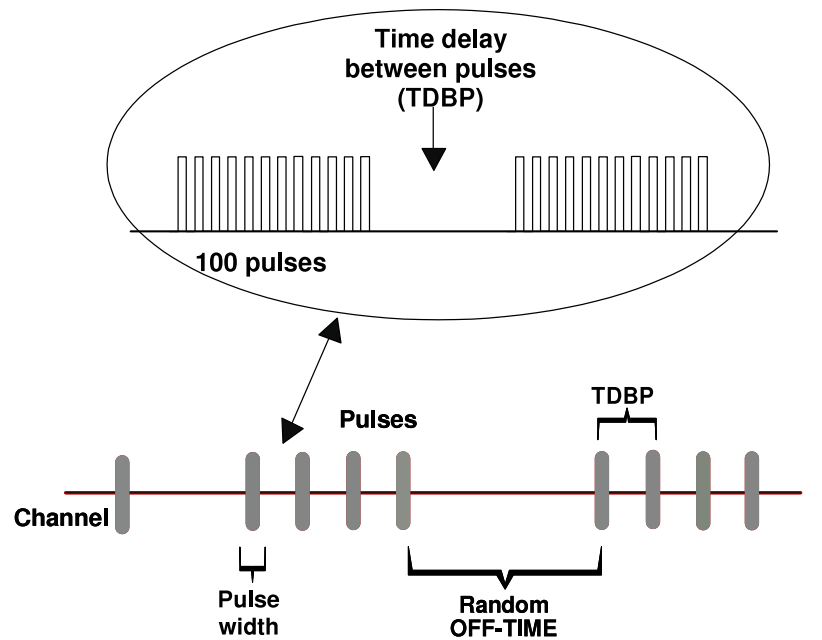

Figure 7. PPM modulator message information scheme.

base. To verify the behaviour of the transmitter, a dynamical linear 3D finite element (FE) model including the transmitter case, the magnets and the PZT element was performed using the commercial software Algore (c) as depicted in figure 5(b) [24]. The mesh consisted principally of bricks, and the boundary conditions of the model were free-free, which were the same as the conditions established in the experiment. The input excitation was $40 \mathrm{~V}$ applied to the faces of the circular PZT crystal, and the attained modal frequency was $44 \mathrm{kHz}$, which differed by $4 \mathrm{kHz}$ from the intended value. The actual transmitter design is shown in figure 6 . The modulator generated 100 square pulses of $40 \mathrm{kHz}$, i.e. $2.5 \mathrm{~ms}$ pulse width. The TDBP pulses were $45 \mathrm{~ms}$ for sensor 1 and $55 \mathrm{~ms}$ for sensor 2. Each smart sensor was programmed into the internal flash memory of the microcontroller. The digital signal was fed to a current booster and then to a high frequency step-up pulse transformer with an input/output ratio of 1:8. This signal was then applied to the actual PZT element. Figure 7 depicts the modulator message information scheme.

\subsection{Receiver design}

The PPM demodulator comprises a $40 \mathrm{kHz}$ ultrasound transducer, a DSP-based digital FIR filter, a microcontroller- based module, which includes an instrumentation amplifier, a threshold comparator, and a USB interface to communicate to a laptop computer. Figure 8 shows the principal elements of the PPM receiver. The DSP module executes a very sharp bandwidth filter using the standard FIR convolution expression [25]

$$
y[n]=\sum_{k=0}^{N-1} h[k] x[n-k]
$$

where the input and output signals are given respectively by $x[n]$ and $y[n]$ and the filter impulse response is denoted by $h[n]$. The coefficients for a particular filter were obtained using the frequency sampling method, whose algorithm is encapsulated by the expression

$$
h[n]=f_{\mathrm{w}}[n] F^{-1}\{H[k]\} \begin{cases}H_{\mathrm{r}}[k]=1, & f_{\mathrm{l}}<k<f_{\mathrm{h}} \\ H_{\mathrm{r}}[k]=0, & \text { elsewhere } \\ H_{\mathrm{i}}[k]=0 & \end{cases}
$$

where $f_{\mathrm{w}}[n]$ denotes a window function and $H[k]$ represents the frequency response of the filter specified in the Fourier domain. For a linear phase filter, the frequency response is determined by setting the real terms $\left(H_{\mathrm{r}}[k]\right)$ to their intended values, denoted by the terms $f_{1}$ and $f_{\mathrm{h}}$, which define the low and high cut-off frequencies of the filter design, and leaving the imaginary terms $H_{\mathrm{i}}[k]$ as zero. The real-time digital filter was implemented with a bandwidth of $2 \mathrm{kHz}$, whose cut-off frequencies were $39 \mathrm{kHz}$ and $41 \mathrm{kHz}$ respectively, i.e. it was designed to operate with the $40 \mathrm{kHz}$ PPM transmitted pulses.

The PPM microcontroller-based demodulator amplifies and converts, in real time, the received and filtered guided wave signals into a set of pulses, using the instrumentation amplifier and the threshold comparator embedded in the microcontroller. The software verifies that the pulses are $40 \mathrm{kHz}$ and discriminates the TDBP, allowing a $6 \mathrm{~ms}$ tolerance due to dispersion; the results are transmitted via USB in ASCII standard to a computer. Collision between modulated transmitted guided waves was minimized by using several off-times in the modulators. 


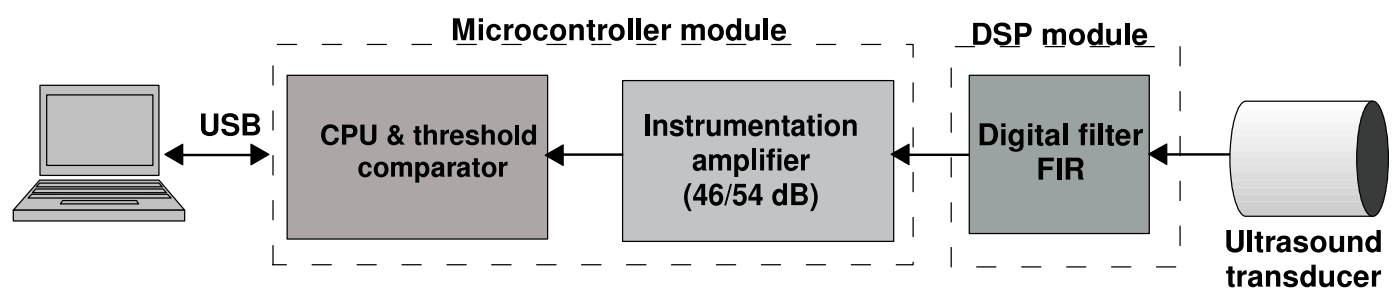

Figure 8. PPM demodulator principal components.

\section{Experimental configuration and results}

A series of experiments were conducted, in dry conditions, in the laboratory and outside the laboratory using tubular steel structures as guided wave communication channels. In the former, a steel tube $1.5 \mathrm{~m}$ in length, $0.27 \mathrm{~m}$ in diameter and $2 \mathrm{~mm}$ in thickness was used. In the latter, two experiments were conducted using structures from an oilrig under construction; first, and mainly, using a tubular steel heliport structure; second, using the base of a deck of an oilrig under construction. The area of the heliport was approximately $15 \mathrm{~m} \times 15 \mathrm{~m}$, and the steel tubes were approximately 0.3 and $0.15 \mathrm{~m}$ in diameter and $4 \mathrm{~mm}$ in thickness.

\subsection{Laboratory results}

Initially the automatic guided waves system was tested in the laboratory to establish that the transmitter modules, receiver and digital filtering systems were operating correctly. In the laboratory setup a PZT ultrasound transducer, acting as receiver and coated with petroleum jelly, was coupled to one end of the pipe. Two smart sensors and PPM modulators were attached to the opposite extreme end and around the tube using strong rare earth magnets. Figure 9 shows this setup. The system was tested by powering the PPM modulators sensor 1 and sensor 2 simultaneously. Figure 10 depicts the signals received from sensor 1 . The lower trace shows the filtered and amplified guided wave signals and the upper trace shows the pulses converted by the microcontroller after the threshold comparator. The system correctly and automatically discriminated between sensor 1 and sensor 2 [26]; however, the distance of transmission was short; hence, a larger scale trial was planned.

\subsection{Field trial results}

Two experiments were conducted at an oilrig construction yard to evaluate the automatic guided wave system instrumentation. In the former, using the heliport of the oilrig under construction, the PZT ultrasound transducer, working as the front end of the demodulator, was coated with petroleum jelly and attached to one extreme of the structure. The smart modulators were also coated with petroleum jelly and attached to the structure via strong permanent magnets and gradually separated from the receiver. The received signals were conveyed via a $2 \mathrm{~m}$ coaxial cable to the instrumentation receiver system. Figure 11 depicts this setup. As described in

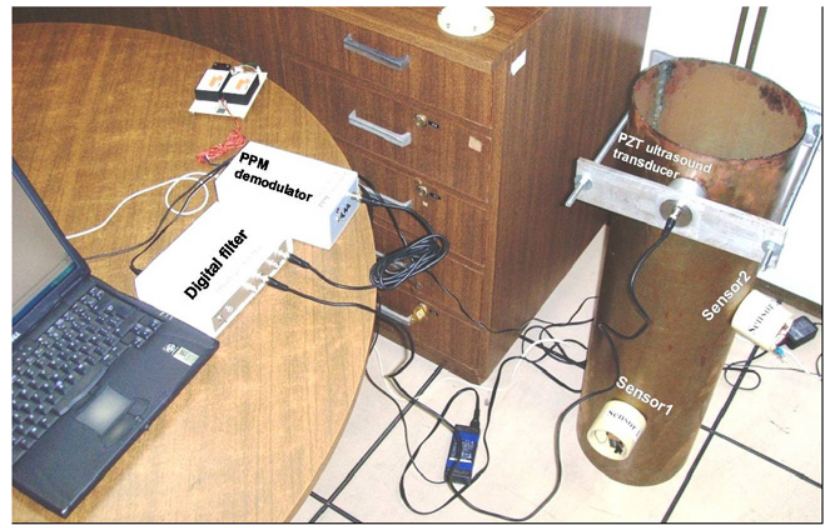

Figure 9. Laboratory experiment setup.

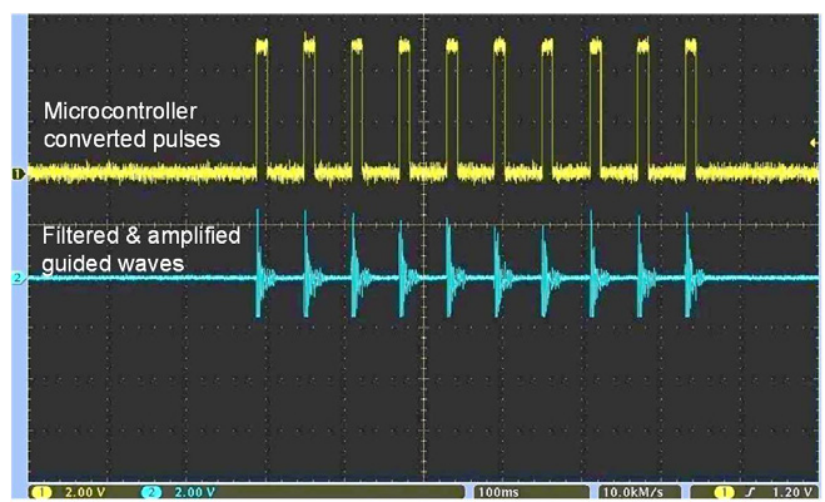

Figure 10. Signals received from sensor 1. Lower signals are the received guided wave pulses filtered and amplified. Upper signals are the pulses converted by the microcontroller after the threshold comparator.

the laboratory tests, the sensors and modulators were powered simultaneously. The smart sensor 2 was placed approximately $1.5 \mathrm{~m}$ from the receiver, but sensor 1 was separated from the transducer receiver at distances of approximately $1,6,15$ and $30 \mathrm{~m}$. Figure 12 depicts the received signals, filtered and amplified, sent by sensor 1 separated from the receiver at 6 and $30 \mathrm{~m}$, before entering the comparator integrated within the microcontroller. The latter experimental setup, using the deck of an oilrig platform under construction, was organized according to the previous trial. However, due to accessibility and limited resources, only the base of the deck was used for the trial. The receiver PZT ultrasound transducer was coated with petroleum jelly and attached to one of the tubes of the structure. The smart modulators were also coated with 

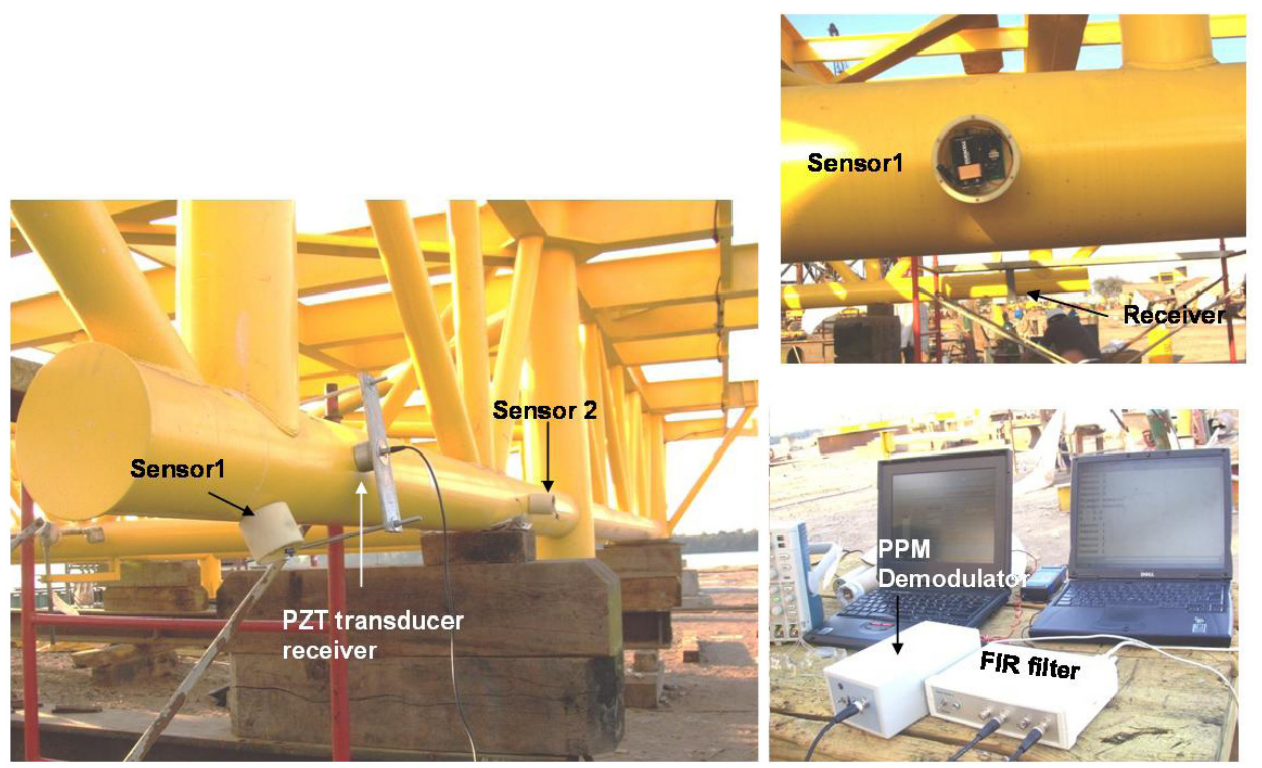

Figure 11. Experiment setup using a tubular heliport structure, its two sensor modulators, its PZT transducer receiver and the instrumentation demodulator system.

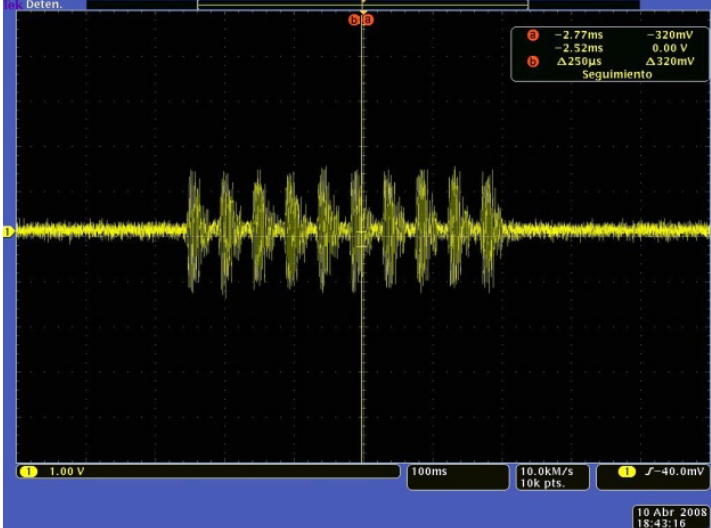

a)

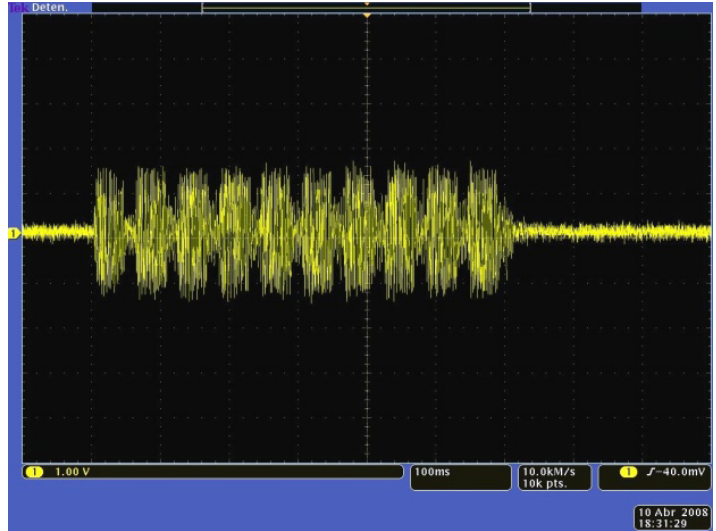

b)

Figure 12. Received guided wave pulses, filtered and amplified using the heliport as communication channel; sensor 1 separated from the receiver by (a) $6 \mathrm{~m}$ and (b) $30 \mathrm{~m}$.

petroleum jelly and attached to the structure and separated from the receiver by approximately $10 \mathrm{~m}$. Figure 13 shows this setup. Correspondingly, figure 14 depicts the received filtered and amplified signals. The signals in both experiments were effectively and automatically identified by the guided wave system, even though they manifested considerable dispersion as the distance increased [27].

\section{Discussion}

Periodic in-service inspections as a continuous process for ensuring the fitness for purpose of sub-sea structures have evolved according to international standards. Underwater close visual inspection of hollow steel crossbeams is increasingly being replaced by FMD techniques, which traditionally make use of divers or ROVs to execute the task. In this application, permanently attached smart sensors that derive benefit from guided ultrasonic waves offer a promising alternative future for FMD systems for remote real-time SHM of sub-sea steel structures in oilrig offshore platforms. The excitation and identification of guided wave signals with sufficient signal to noise ratio has been achieved, in a visual manner at the monitoring receiver at surface level, in previous experiments carried out by the authors in a $7 \mathrm{~m}$ long tubular structure flooded and immersed in seawater. In this work, however, the emphasis is in the automatic detection of guided wave pulses using PPM for encoding information of different transmitters. The trials carried out in this work used instrumentation designed specifically for the purpose of evaluating a novel automatic guided wave system that exploits steel pipes as the communication medium. Although the experiments inside and outside the laboratory were performed in dry conditions, the results substantiate that PPM can be employed to transmit guided wave encoded information 

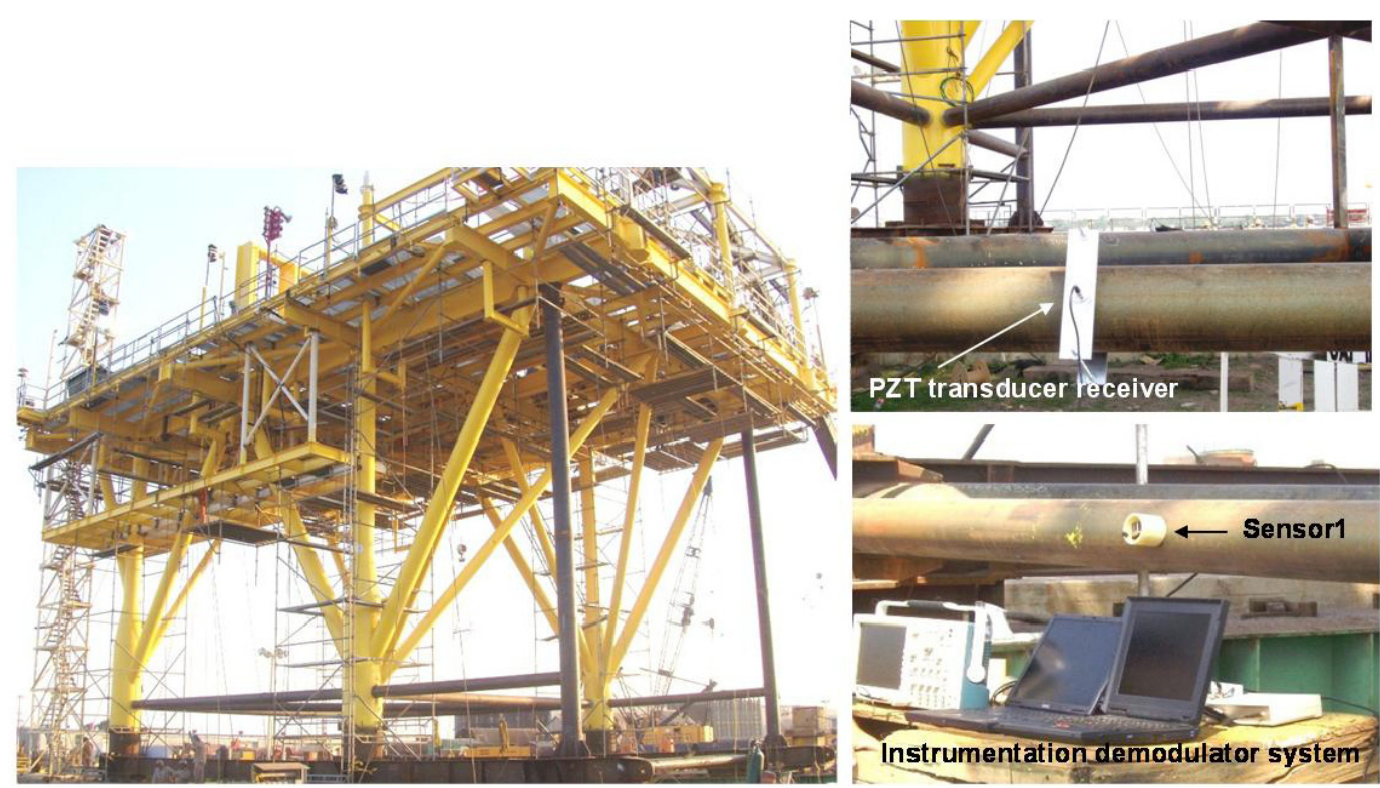

Figure 13. Experiment setup using the base deck of an oilrig under construction, its sensor modulator, its PZT transducer receiver and the instrumentation demodulator system.

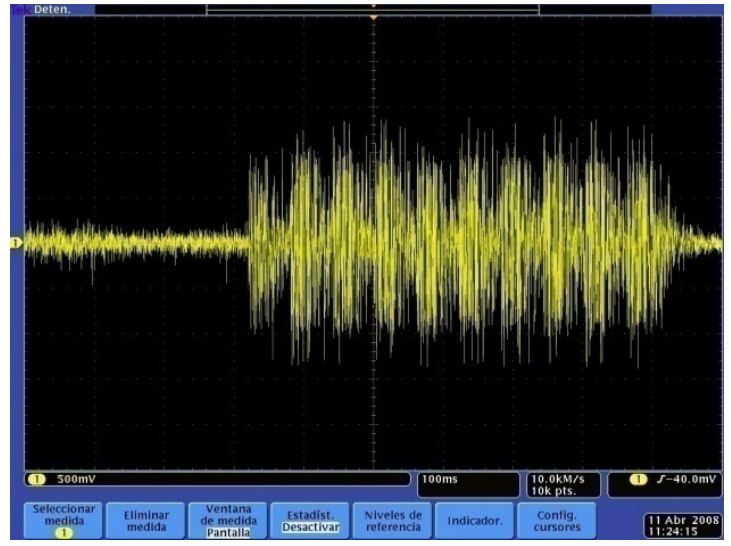

a)

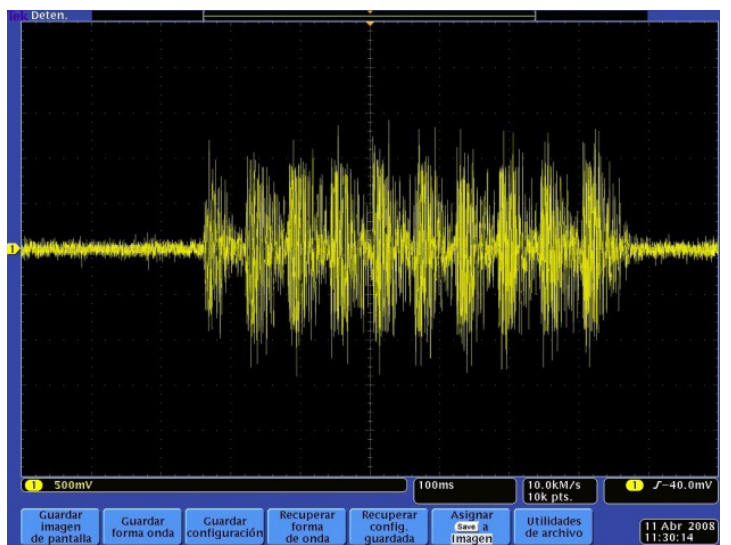

b)

Figure 14. Received guided wave pulses, filtered and amplified, using the base deck of the oilrig; sensors separated from the receiver by $10 \mathrm{~m}$, (a) PPM pulses sent by sensor 1, (b) PPM pulses sent by sensor 2.

through pipe specimens of greater length than the $30 \mathrm{~m}$ achieved in these tests. These results could be scaled in such a manner that would allow similar tests to be conducted on full size oilrigs, since the power of the transmitter, $9 \mathrm{~V}$, and the gain of the receiver, $54 \mathrm{~dB}$, were relatively low; hence, a carefully designed system could be constructed to detect seawater flooding in structures in excess of $100 \mathrm{~m}$ in length.

\section{Conclusion}

The feasibility of using an automatic PPM guided wave system for the purpose of detecting flooding in sub-sea members of oil rigs has been designed, implemented and evaluated using oilrig steel structures. Results show that by exploiting the waveguide effect of steel pipes, which act as communication channels, successful transmission and reception of PPM encoded information has been achieved this corroborates theoretical predictions yielded by the software Disperse. Although the tests were conducted in dry conditions, the ability to detect dispersive guided wave energy packets, provided sufficient SNR, has been demonstrated. These results are very encouraging, taking the authors to the next stage of this work, which is to attach permanent smart sensors in a real offshore oilrig under construction for subsequent experimental monitoring.

\section{Acknowledgments}

The authors wish to express their thanks to the Instituto de Investigaciones Eléctricas (IIE) project 13403 and CONACYT for financially supporting this work and to SWECOMEX for allowing the testing of the system. 


\section{References}

[1] Kessler S S and Chang F K 1998 Structural Health Monitoring: Current Status and Perspectives (Lancaster, PA: CRC Press)

[2] Kessler S S 2002 Piezoelectric-based in-situ damage detection of composite materials for structural health monitoring systems PhD Thesis Massachusetts Institute of Technology

[3] Kessler S S 2005 Certifying a structural health monitoring system: characterizing durability, reliability and longevity Proc. 1st Int. Forum on Integrated Systems Health Engineering and Management in Aerospace (Napa, CA, Nov. 2005)

[4] ISO19902 2007 Petroleum and Natural Gas Industries-Fixed Steel Offshore Structures

[5] Marsh L M, Sun C, Pillai B K and Viana L 2005 Data recovery for pulse telemetry using pulse position modulation Patent No. 6,963,290

[6] Burdekin F M, Talai-faz B, Brennan F P and Dover W D 2001 Experimental validation of the ultimate strength of brace members with circumferential cracks Offshore Technology Reports 081 UMIST and University College London for the Health and Safety Executive

[7] Mijarez R, Gaydecki P and Burdekin M 2007 Flood member detection for real-time structural health monitoring of sub-sea structures of offshore steel oilrigs Smart Mater. Struct. 16 1857-69

[8] Mijarez R, Gaydecki P and Burdekin M 2005 An axisymmetric guided wave encoded system for flood detection of oil rig cross-beams Meas. Sci. Technol. $162265-74$

[9] Rose J L 2004 Ultrasonic guided waves in structural health monitoring Key Engineering Materials vol 270-273 (Switzerland: Trans Tech Publications) pp 14-21

[10] Cawley P and Simonetti F 2005 Structural health monitoring using guided waves-potential and challenges The 5th Int. of Workshop Structural Health Monitoring (Stanford University) pp 503-10

[11] Gazis D C 1959 Three dimensional investigation of the propagation of waves in hollow circular cylinders I. Analytical foundation J. Acoust. Soc. Am. 31 568-73

[12] Silk M G and Bainton K F 1979 The propagation in metal tubing of ultrasonic wave modes equivalent to Lamb waves Ultrasonics 17 11-9
[13] Pavlakovic B and Lowe M 2003 Disperse User Manual: A System for Generating Dispersion Curves (London: Imperial College) Copyright B Pavlakovic, M Lowe (c) 2003

[14] Cawley P, Lowe M J S, Alleyne D N, Pavlakovic B and Wilcox P 2003 Practical long range guided wave testing: applications to pipes and rail Mater. Eval. 61 66-74

[15] Rose J L 1999 Ultrasonic Waves in Solid Media (Cambridge: Cambridge University Press)

[16] Shin H J and Rose J L 1999 Guided waves by axisymmetric and non-axisymmetric surface loading on hollow cylinders Ultrasonics 37 355-63

[17] Cawley P and Alleyne D 1996 The use of Lamb waves for the long range inspection of large structures Ultrasonics 34 287-90

[18] Mijarez R, Gaydecki P and Burdekin M 2005 Continuous monitoring guided wave encoded sensor for flood detection of oil rig leg crossbeams Insight 47 748-50

[19] Pollakowski M and Ermert H 1994 Chirp signal matching and signal power optimization in pulse-echo mode ultrasonic nondestructive testing IEEE Trans. Ultrason. Ferroelectr. Freq. Control 41 655-9

[20] McAulay R J 1968 Optimal control techniques applied to PPM signal design Inform. Control 12 221-35

[21] Azmia P et al 2004 An efficient method for demodulating PPM signals based on Reed-Solomon decoding algorithm Signal Process. 84 1823-36

[22] Haykin S 2002 Communication Systems (New York: Wiley)

[23] Wonzencraft J 1965 Principles of Communication Engineering (New York: Wiley)

[24] Algor@ V20.4 2007 Instructor Manual

[25] Gaydecki P 2004 Foundations of Digital Signal Processing: Theory, Algorithms and Hardware Design (London: The Institute of Electrical Engineers)

[26] Mijarez R, Martinez F and Gaydecki P 2008 An automatic guided wave pulse position modulation system using steelpipes as a communication channel for flood detection in steel offshoreoilrigs World Conf. on Nondestructive Testing WCNDT (Shanghai, Oct. 2008)

[27] Mijarez R, Gaydecki P and Burdekin M 2005 Continuous structural health monitoring guided wave PPM system using steel pipes as communication channel for flood detection in steel offshore oilrigs Annual Review of Progress in Quantitative NDE (Chicago, IL, July 2008) ed D E Chimenti and R B Thompson (New York: Plenum) pp 1014-21, QNDE 2008 